\title{
LA DOCENCIA A TRAVÉS DE LA INVESTIGACIÓN-ACCIÓN
}

\author{
Esperanza Bausela Herreras \\ Becaria de investigación de la Universidad de León, España
}

\section{INTRODUCCIÓN}

La investigación acción, es un término acuñado y desarrollado por Kurt Lewin en varías de sus investigaciones (Lewin, 1973), actualmente, es utilizado con diversos enfoques y perspectivas, depende de la problemática a abordar.

Es una forma de entender la enseñanza, no sólo de investigar sobre ella. La investigación - acción supone entender la enseñanza como un proceso de investigación, un proceso de continua búsqueda. Conlleva entender el oficio docente, integrando la reflexión y el trabajo intelectual en el análisis de las experiencias que se realizan, como un elemento esencial de lo que constituye la propia actividad educativa. Los problemas guían la acción, pero lo fundamental en la investigación - acción es la exploración reflexiva que el profesional hace de su practica, no tanto por su contribución a la resolución de problemas, como por su capacidad para que cada profesional reflexione sobre su propia práctica, la planifique y sea capaz de introducir mejoras progresivas. En general, la investigación - acción cooperativa constituye una vía de reflexiones sistemática sobre la práctica con el fin de optimizar los procesos de enseñanza - aprendizaje.

\section{EVOLUCIÓN HISTÓRICA}

Elliot es el principal representante de la investigación acción desde un enfoque interpretativo "El propósito de la investigación - acción consiste en profundizarla comprensión del profesor (diagnóstico) de su problema. Por tanto, adopta una postura exploratoria frente a cualesquiera definiciones iniciales de su propia situación que el profesor pueda mantener...La investigación acción interpreta lo que ocurre desde el punto de vista de quienes actúan e interactúan en la situación problema, por ejemplo, profesores y alumnos, profesores y director". (Elliot, 1993).

Si tuviéramos que señalar los hitos más significativos en la historia de la investigación - acción podemos destacar los tres siguientes, según Contreras (1994);

- El primero es el trabajo de Kurt Lewin $(1946,1952)$. Aunque la idea de investigación - acción ya la habían utilizado otros autores anteriormente, fue Lewin, en los años 40, en Estados Unidos, quien le dio entidad al intentar establecer una forma de investigación que no se limitara, según su propia expresión, a producir libros, sino que integrara la experimentación científica con la acción social. Definió el trabajo de investigación - acción como un proceso cíclico de exploración, actuación y valoración de resultados.

- No es hasta comienzos de los años 70 y en Gran Bretaña, Lawrence Stenhouse y de John Elliott. Pero para ellos ya no significa una técnica de investigación para ocasionar cambios, sino la convicción de que las ideas educativas sólo pueden expresar su auténtico valor cuando se intenta traducirlas a la práctica, y esto sólo pueden hacerlo los enseñantes investigando con su práctica y con las ideas con las que intentan guiarse (Stenhouse, 1984). Tal y como la define 
Elliott (1993: 88), la investigación - acción se entiende como «el estudio de una situación social para tratar de mejorar la calidad de la acción en la misma».

- Stephen Kemmis junto con Wilfred Carr y el equipo de la Universidad de Deakin, en Australia, desde comienzos de los años 80, buscan una reconceptualización de la investigación - acción. Consideran que ésta no puede entenderse como un proceso de transformación de las prácticas individuales del profesorado, sino como un proceso de cambio social que se emprende colectivamente.

\section{FINALIDAD}

Gollete y Lesgard - Hervert (1988) identifican tres funciones y finalidades básicas; (i) investigación, (ii) acción y (iii) formulación / perfeccionamiento. Afirman que este tipo de investigación beneficia simultáneamente el desarrollo de destrezas, la expansión de la teoría y la resolución de problemas.

\begin{tabular}{l}
\hline FORMACIÓN PROFESIONAL \\
- Genera actitudes de crítica y \\
renovación profesional \\
- Favorece el cambio y la \\
trasformación de la acción \\
- Supone la participación y la \\
modificación del entorno
\end{tabular}

DIMENSIÓN

DIMENSIÓN

\section{ASPECTOS FORMATIVOS}

- Trasformación de actitudes y comportamientos

- Aprendizaje activo,

construcción del saber

- Posibilita el desarrollo personal
PARTICIPACIÓN SOCIAL

- Refuerza la concienciación de los sujetos en el proceso social

- Insta a los sujetos a la participación en el desarrollo social

SOCIAL

OBJETIVOS

FORMATIVOS DE LA INVESTIGACIÓN

PARTICIPATIVA

Figura 1 Posibilidades formativas de la investigación - acción participativa

(Tomado de Colás Bravo, 1994: 295)

\section{CARACTERÍSTICAS}

La investigación - acción se presenta como una metodología de investigación orientada hacia el cambio educativo y se caracteriza entre otras cuestiones por ser un proceso que como señalan Kemmis y MacTaggart (1988); (i) Se construye desde y para la práctica, (ii) pretende mejorar la práctica a través de su trasformación, al mismo tiempo que procura comprenderla, (iii) demanda la participación de los sujetos en la mejora de sus propias prácticas, (iv) exige una actuación grupal por laque los sujetos implicados colaboran coordinadamente en todas las fases del proceso de investigación, (v) implica la realización de análisis crítico de las situaciones y (vi) se configura como una espiral de ciclos de planificación, acción, observación y reflexión.

Entre los puntos clave de la investigación - acción, Kemmis y Mctaggart (1988) destacan la mejora de la educación mediante su cambio, y aprender a partir de la consecuencias de los cambios y la 
planificación, acción, reflexión nos permite dar una justificación razonada de nuestra labor educativa ante otras persona porque podemos mostrar de qué modo las pruebas que hemos obtenido y la reflexión crítica que hemos llevado a cabo nos han ayudado a crear una argumentación desarrollada, comprobada y examinada críticamente a favor de lo que hacemos.

A estas características debemos unir las siguientes; (i) No se puede reducir al aula, porque la práctica docente tampoco está limitada ni reducida a ella. Investigar nos lleva a cambiar la forma de entender la práctica: qué damos por sentado, qué cuestionamos, qué nos parece natural o inevitable (o por encima de nuestras posibilidades o responsabilidades), y qué nos parece discutible y necesario transformar, y en lo que nos sentimos comprometidos. (ii) Es una forma por la cual el profesorado puede reconstruir su conocimiento profesional como parte del proceso de constitución de discursos públicos unidos a la práctica, y sus problemas y necesidades. (iii) No puede ser nunca una tarea individual. Debe ser, por el contrario, un trabajo cooperativo. Cualquier tarea de investigación requiere un contexto social de intercambio, discusión y contrastación. Este tipo de contextos es el que hace posible la elaboración y reconstrucción de un conocimiento profesional no privado y secreto, sino en diálogo con otras voces y con otros conocimientos. (iv) Como cualquier planteamiento que trate de defender una práctica docente reflexiva, investigadora, de colaboración con colegas, necesita de unas condiciones laborales que la hagan posible. (v) Es una tarea que consume tiempo, porque lo consume la discusión con colegas, la planificación conjunta de tareas, la recogida de información, su análisis.

La investigación - acción no se limita a someter a prueba determinadas hipótesis o a utilizar dato para llegar a conclusiones. La investigación - acción es un proceso, que sigue una evolución sistemática, y cambia tanto al investigador como las situaciones en las que éste actúa. Pérez Serrano (1994) esquematiza los rasgos que definen la investigación acción en la siguiente figura 2;

\section{ACCIÓN}

Unión teoría y práctica Mejora la acción Problemas prácticos Protagonismo del práctico

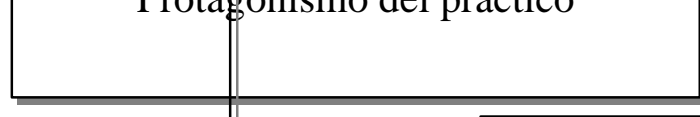

CAMBIO

\section{INVESTIGACIÓN}

Cambio en colaboración

Democratización del proceso

Función crítica

Función de comunicación

Acción como cambio social

Finalidad de formación

Figura 2 Rasgos que definen la investigación - acción (Pérez Serrano, 1997: 75).

Algunas ventajas de implicarse en procesos de investigación - acción se relacionan con un aumento de la autoestima profesional, la disminución del aislamiento profesional y el refuerzo de la motivación profesional. Permite que los profesionales investiguen, y forme un profesional reflexivo.

\section{TIPOLOGÍAS}


Las propuestas que han proliferado para catalogar las distintas modalidades de la investigación acción se basan en diversos criterios; principios ideológicos (Carr y Kemmis, 1988), objetos científicos y niveles de participación (Desroche, 1981). Nos detendremos en el propuesto por Grundy $(1982,1991)$ quien ha señalado tres modelos básicos de investigación - acción: el técnico, el práctico y el crítico o emancipador En la Figura 3 se describen ambas modalidades.

\begin{tabular}{|l|l|l|l|l|}
\hline MODALIDADES & \multicolumn{1}{|c|}{$\begin{array}{c}\text { TIPO DE } \\
\text { CONOCIMIENTO } \\
\text { QUE GENERAN }\end{array}$} & OBJETIVOS & $\begin{array}{l}\text { FORMAS } \\
\text { DE } \\
\text { ACCIÓN }\end{array}$ & $\begin{array}{c}\text { NIVEL DE } \\
\text { PARTICIPACIÓN }\end{array}$ \\
\hline I/A TÉCNICA & Técnico /explicativo & $\begin{array}{l}\text { Mejorar las } \\
\text { acciones y la } \\
\text { eficacia del } \\
\text { sistema }\end{array}$ & $\begin{array}{l}\text { Sobre la } \\
\text { acción }\end{array}$ & $\begin{array}{l}\text { Cooptación } \\
\text { Designación }\end{array}$ \\
\hline I/A PRÁCTICA & Práctico & $\begin{array}{l}\text { Comprender } \\
\text { la realidad }\end{array}$ & $\begin{array}{l}\text { Para la } \\
\text { acción }\end{array}$ & Cooperación \\
\hline I/A CRÍTICA & Emancipativo & $\begin{array}{l}\text { Participar en } \\
\text { la } \\
\text { trasformación } \\
\text { social }\end{array}$ & $\begin{array}{l}\text { Por la } \\
\text { acción }\end{array}$ & Implicación \\
\hline
\end{tabular}

Figura 3 Modalidades de investigación - acción: criterios de identificación (pp. 293).

- El primero, el técnico, tendrá que ver con aquellos procesos guiados por expertos en los que los prácticos ejecutan la investigación diseñada por aquellos y dirigida a la obtención de resultados ya prefijados, con una clara preocupación productivista o eficientista.

- El segundo, el práctico, son procesos de investigación - acción dirigidos a la realización de aquellos valores intrínsecos a la práctica educativa, por lo que suponen un proceso de indagación y reflexión de la práctica a la luz de sus fines y, viceversa, de los fines o valores a la luz de los acontecimientos prácticos. Es la perspectiva que representa el trabajo de Elliott y Stenhouse.

- El tercer modelo, el crítico, parte de la idea de que no siempre es posible la realización de lo que supone el modelo práctico debido a las restricciones institucionales e ideológicas. Por esta razón, no es suficiente con plantearse la práctica particular, sino que es necesario plantearse, además, la transformación de estas estructuras restrictivas, para lo cual es necesario acudir a fuentes teóricas críticas que sirvan de soporte a esta toma de conciencia de las limitaciones de la práctica. Ésta es la perspectiva que representa el trabajo de Carr y Kemmis, y de la propia Grundy.

No obstante, con objeto de clarificar la clasificación de Grundy, (Contreras, 1994) es necesario hacer algunas matizaciones;

- En primer lugar, el modelo técnico, si bien representa una clara tendencia respecto a la proliferación de experiencias que se presentan como de investigación - acción, la verdad es que, si nos atenemos a los rasgos anteriormente expuestos, no parece que pueda considerarse como tal investigación - acción. Como vemos, no pone en cuestión la visión técnica de relación entre las actuaciones y sus pretensiones, y convierte a los prácticos en realizadores de prescripciones ajenas. Sin embargo, es importante poderla tener en cuenta como forma de llamar la atención respecto a determinados vicios instalados, o también respecto a 
determinadas pretensiones de que el profesorado investigue sobre su práctica sin concederle la oportunidad de que cuestione las prescripciones curriculares o las condiciones institucionales en que se desenvuelve la docencia.

- En segundo lugar, aunque se presente al modelo crítico como una superación indiscutible del práctico, debe tenerse en cuenta que la cosa es más complicada que eso, ya que, a su vez, los defensores de este último han discutido algunos aspectos en el crítico, como puede ser el peligro de hacer depender al profesorado de las perspectivas académicas, dada la importancia que en este modelo se le concede a la teoría. Lo que sí podemos afirmar es que ambos modelos representan posiciones ideológicas y estratégicas diferentes respecto a la forma en que deben producirse las transformaciones sociales y el papel que puede desempeñar la investigación - acción en ello.

\section{PROCESO}

De forma genérica podemos decir que la investigación acción se desarrolla siguiendo un modelo en espiral en ciclos sucesivos que incluyen diagnóstico, planificación, acción, observación y reflexión evaluación. El proceso de investigación acción es descrito con matizaciones diferentes según autores, variando en cuanto a su complejidad (Lewin, Kemmis, MacTaggart, Ander Egg, Elliot...). La figura 4 nos muestra las principales fases;

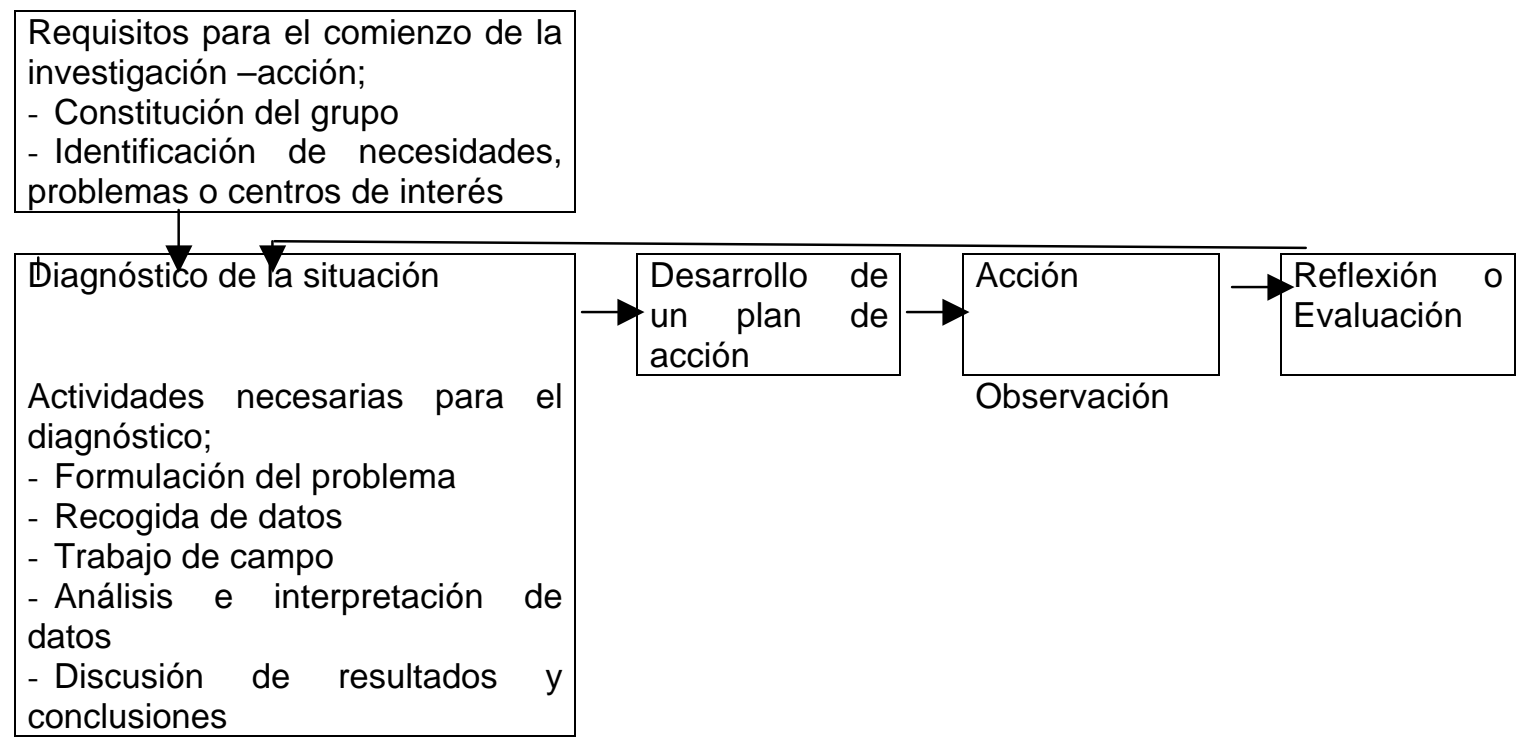

Figura 4 Proceso de investigación - acción (Tomado de Colás Bravo, 1994: 297).

Como podemos observar, todo este proceso se resume en cuatro fases (Kemmis McTaggart, 1988): (i) Diagnóstico y reconocimiento de la situación inicial.(ii) Desarrollo de un plan de acción, críticamente informado, para mejorar aquello que ya está ocurriendo. (iii) Actuación para poner el plan en práctica y la observación de sus efectos en el contexto que tiene lugar. (iv) La reflexión en torno a los efectos como base para una nueva planificación.

Según Rincón y Rincón (2000) en general, el planteamiento de un proceso de mejora en el ámbito educativo suele basarse en la actuación de equipos docentes que se constituyen en grupos de revisión y mejora y revisiones sucesivas. El proceso propuesto por Rincón y Rincón (2000) se puede esquematizar en la siguiente figura 5 . 
PROCESO GENERAL DE REVISIÓN Y MEJORA

\begin{tabular}{|l|l|l|l|}
\hline \multicolumn{1}{|c|}{ FASES } & \multicolumn{1}{|c|}{ A) Objetivos } & B) Recogida/análisis datos & \multicolumn{1}{c|}{ C) Informe } \\
\hline $\begin{array}{l}\text { 1. Plantea- } \\
\text { miento }\end{array}$ & $\begin{array}{l}\text { Concienciar } \\
\text { necesidad de } \\
\text { optimizar. }\end{array}$ & Sesiones grupales. & $\begin{array}{l}\text { Acuerdos y } \\
\text { decisiones. }\end{array}$ \\
\hline $\begin{array}{l}\text { 2. Revisión } \\
\text { global }\end{array}$ & $\begin{array}{l}\text { Priorizar áreas de } \\
\text { mejora asumibles. }\end{array}$ & $\begin{array}{l}\text { Lluvia de ideas. Listas. } \\
\text { Grupo nominal. }\end{array}$ & Áreas prioritarias. \\
$\begin{array}{l}\text { 3. Revisión } \\
\text { específica }\end{array}$ & Diagnosticar. & $\begin{array}{l}\text { Listas. Guía revisión. Perfiles. } \\
\text { Mapas. Diagramas. } \\
\text { Indicadores. }\end{array}$ & $\begin{array}{l}\text { Problemas. } \\
\text { Necesidades. } \\
\text { Causas. }\end{array}$ \\
\hline $\begin{array}{l}\text { 4. Planificar } \\
\text { mejoras }\end{array}$ & $\begin{array}{l}\text { Elaborar plan de } \\
\text { actuación. }\end{array}$ & $\begin{array}{l}\text { Lluvia de ideas. Mapas. } \\
\text { Diagramas. }\end{array}$ & $\begin{array}{l}\text { Qué se hará, cuándo, } \\
\text { cómo, con qué, quién. }\end{array}$ \\
\hline $\begin{array}{l}\text { 5. Aplicar } \\
\text { mejoras }\end{array}$ & $\begin{array}{l}\text { Implantar plan de } \\
\text { actuación. }\end{array}$ & $\begin{array}{l}\text { Listas. Guía revisión. Perfiles. } \\
\text { Mapas. Diagramas }\end{array}$ & $\begin{array}{l}\text { Material referencial. } \\
\text { Evidencias. }\end{array}$ \\
\hline $\begin{array}{l}\text { 6. Revisar } \\
\text { mejoras }\end{array}$ & $\begin{array}{l}\text { Valorar plan de } \\
\text { actuación. }\end{array}$ & $\begin{array}{l}\text { Listas. Guía revisión. Perfiles. } \\
\text { Mapas. Diagramas. } \\
\text { Indicadores. }\end{array}$ & $\begin{array}{l}\text { Aspectos positivos, } \\
\text { negativos. } \\
\text { Dificultades. }\end{array}$ \\
\hline $\begin{array}{l}\text { 7. Planificar } \\
\text { nuevas } \\
\text { mejoras }\end{array}$ & $\begin{array}{l}\text { Institucionalizar. } \\
\text { Optimizar plan de } \\
\text { acción. Nueva área. }\end{array}$ & $\begin{array}{l}\text { Lluvia de ideas. Mapas. } \\
\text { Diagramas. }\end{array}$ & $\begin{array}{l}\text { Qué se hará, cuándo, } \\
\text { cómo, con qué, quién. }\end{array}$ \\
\hline
\end{tabular}

Figura 5 Proceso general de revisión y mejora (tomado de Rincón y Rincón, 2000)

\section{INSTRUMENTOS DE RECOGIDA DE DATOS}

La recogida de información se efectuará utilizando diversos instrumentos, previstos en el diseño de investigación del propio plan de trabajo. Para la recogida de información se han utilizado tres instrumentos básicos: los estudios cuantitativos, las observaciones y los diarios. La utilización de estos tres instrumentos básicos de recogida de información no excluye el posible uso de otros complementarios y habituales en los procesos de investigación-acción: análisis de documentos, datos fotográficos, grabaciones en audio y vídeo (con sus correspondientes transcripciones), entrevistas, encuestas de opinión, etc. Así, por ejemplo, de una entrevista, más o menos estructurada, se pueden extraer datos cuantitativos, observaciones e impresiones para el diario. Un detalle específico de técnicas puede verse consultando Kemmis y Mctaggart (1988). Rincón (1997) proponen recoger información de diferentes ámbitos con ayuda de; observación, entrevista y análisis de documentos (figura 6).

\begin{tabular}{|c|c|c|c|c|}
\hline \multirow{2}{*}{\multicolumn{2}{|c|}{$\begin{array}{l}\text { ÁMBITO POR EVALUAR } \\
\text { DATOS SOBRE } \\
\end{array}$}} & \multicolumn{3}{|c|}{ TÉCNICAS DE OBTENCIÓN DE INFORMACIÓN } \\
\hline & & OBSERVACIÓN & ENTREVISTAS & DOCUMENTOS \\
\hline $\begin{array}{l}\text { Revisión Inicial } \\
\text { y específica }\end{array}$ & Institución global & $\begin{array}{ll}\text { Listas } & \text { de } \\
\text { comprobación } \\
\text { Escalas de } \\
\text { estimación }\end{array}$ & $\begin{array}{ll}\text { Grupo } & \text { de } \\
\text { discusión } & \\
\text { Sondeo } & \text { de } \\
\text { problemas } & \end{array}$ & $\begin{array}{ll}\text { Actas } \\
\text { Circulares } \\
\text { Inventario } \\
\text { recursos } \\
\text { Indicadores } \\
\end{array}$ \\
\hline \multirow{2}{*}{$\begin{array}{l}\text { Planificación } \\
\text { procesos } \\
\text { optimización }\end{array}$} & Objetivos & & \multirow{2}{*}{$\begin{array}{l}\text { Grupo nominal } \\
\text { Sistema de ideas } \\
\text { clave } \\
\text { Campo de } \\
\text { fuerzas }\end{array}$} & $\begin{array}{l}\text { Matriz } \\
\text { decisiones }\end{array}$ \\
\hline & $\begin{array}{l}\text { Acciones } \\
\text { Previstas }\end{array}$ & & & \\
\hline \multirow[t]{2}{*}{$\begin{array}{l}\text { Seguimiento de } \\
\text { la aplicación y } \\
\text { efectos }\end{array}$} & $\begin{array}{l}\text { Actividades que } \\
\text { se realizan }\end{array}$ & $\begin{array}{ll}\text { Listas } & \text { de } \\
\text { comprobación } & \\
\text { Escalas de } \\
\text { estimación }\end{array}$ & & $\begin{array}{l}\text { Programas } \\
\text { Diarios }\end{array}$ \\
\hline & Dificultades & & $\begin{array}{l}\text { Sondeo } \\
\text { problemas }\end{array}$ & Diarios \\
\hline
\end{tabular}




\begin{tabular}{|c|c|c|c|c|}
\hline & $\begin{array}{l}\text { Satisfacción } \\
\text { participantes }\end{array}$ & $\begin{array}{ll}\text { Listas } & \text { de } \\
\text { comprobación } & \\
\text { Escalas } & \text { de } \\
\text { estimación } & \end{array}$ & $\begin{array}{ll}\text { Grupo } & \text { de } \\
\text { discusión } & \end{array}$ & \\
\hline \multirow[t]{2}{*}{$\begin{array}{l}\text { Incidencia en la } \\
\text { institución }\end{array}$} & Trasferencia & \multirow{2}{*}{$\begin{array}{ll}\text { Listas } & \text { de } \\
\text { comprobación } & \\
\text { Escalas } & \text { de } \\
\text { estimación } & \end{array}$} & & $\begin{array}{l}\text { Proyectos } \\
\text { Informes }\end{array}$ \\
\hline & Cambios & & $\begin{array}{ll}\text { Grupo } & \text { de } \\
\text { discusión } & \\
\text { Campos } & \text { de } \\
\text { fuerza } & \end{array}$ & Indicadores \\
\hline
\end{tabular}

Figura 6 Ejemplo de diseño con algunos ámbitos y técnicas de recogida de información (tomado de Rincón, 1997: 83).

\section{PRINCIPIOS ÉTICOS}

Dado que la investigación - acción se lleva a cabo en medios humanos donde están implicadas otras personas (alumnado u otras) y que el tipo de datos que pretendemos obtener puede significar manejar información sobre las personas o sus contextos y circunstancias, cuyo uso inadecuado pudiera ser lesivo para las mismas, es necesario cuidar tanto los modos por los cuales tenemos acceso a la información, como la interpretación que de ella hacemos y el uso público que le damos. Esto justifica la defensa de unos principios éticos (véase figura 7) que deben defenderse siempre por encima de cualquier interés investigador.

1. Todas las personas e instancias relevantes para el caso deben ser consultadas y deben obtenerse los consentimientos precisos.

2. Deben obtenerse permisos para realizar observaciones (salvo cuando se trate de la propia clase) o examinar documentos que se elaboran con otros propósitos diferentes al de la investigación y que no sean públicos.

3. Cuando la realización del proyecto requiera de la implicación activa de otras partes, todos los participantes deberán entonces tener oportunidad de influir en el desarrollo del mismo, así como debe respetarse el deseo de quienes no deseen hacerlo.

4. El trabajo debe permanecer visible y abierto a las sugerencias de otros.

5. Cualquier descripción del trabajo o del punto de vista de otros debe ser negociado con ellos antes de hacerse público.

6. El alumnado tiene los mismos derechos que el profesorado, o cualesquiera otros implicados, respecto a los datos que proceden de ellos. En concreto, debe negociarse con los alumnos y alumnas las interpretaciones de los datos que procedan de ellos y obtenerse su autorización para hacer uso público de los mismos.

7. En los informes públicos de la investigación, debe mantenerse el anonimato de las personas que participan en ella, así como de las instituciones implicadas, a no ser que haya deseo en contrario de los interesados y autorización para ello. En todo caso, debe mantenerse el anonimato del alumnado.

8. Todos los principios éticos que se establezcan deben ser conocidos previamente por los afectados y acordados con ellos, así como los términos de su uso. 
Figura 7 Principios éticos de la investigación - acción (Fuente: Kemmis y McTaggart, 1988; Winter, 1989; Altrichter y otros, 1993).

\section{ALGUNAS APLICACIONES EN EL ÁMBITO EDUCATIVO}

La investigación - acción se revela como uno de los modelos de investigación más adecuados para fomentar la calidad de la enseñanza e impulsar la figura del profesional investigador, reflexivo y en continua formación permanente (Rincón, 1997).

Entre los diversos campos de aplicación de la investigación -acción en educación tenemos que destacar la aplicación para la evaluación de centros, aprendizaje, instituciones... De la revisión bibliográfica podemos reseñar los siguientes ejemplos

Son diversos los ejemplos a los que podemos hacer referencias sobre estudios basados en esta metodología, la cual es considerada como una vía para el cambio (Bartolomé Pina, 1992). Tiene como fin último mejorar la realidad vivida, busca evaluar para cambiar la realidad desde la realidad misma (Kemmis \& MacTaggart, 1988, Kemmis, 1988). A continuación enunciaremos y comentaremos brevemente algunos de ellos.

López Górriz (1993) nos proporciona un proyecto de investigación - acción llevado a cabo por siete profesores de la zona de Aljirafe de Sevilla y por un equipo de investigación de la Universidad de Sevilla. El proyecto tenía una doble finalidad; (i) ayudar al profesorado a adquirir capacidades investigadoras que les permitan hacer un diagnóstico preciso de la problemática educativa en su aula y (ii) ofrecer alternativas de acción e introducir una mejora educativa e innovación curricular, que beneficie al adulto, así como su evaluación.

Lapuebla (1988) nos proporciona la experiencia de proyecto educativo que tiene como uno de sus tres grandes objetivos la transformación de los procesos de aprendizajes, de modo que los alumnos sean sujetos activos de su propia formación y no simples elementos pasivos; la actividad debe pasar por el conocimiento de las características del individuo, sus necesidades, actitudes y capacidades.

Buendía y Salmerón (1994) desarrollan un estudio cuyo objetivo específico es conocer la actitud de los profesores hacia la investigación educativa y la posible tendencia al cambio de actitudes una vez finalizado un curso de formación y participación en un proyecto de investigación cooperativa.

Perelló, Membrive y Vives (1995) realizaron una experiencia con un grupo de alumnos de primero y segundo de preescolar y con la colaboración de los padres, en el Colegio de Capdepera (Mallorca) con objeto de cambiar los hábitos alimentarios de los niños los cuales a menudo no son los más adecuados dietéticamente.

Concluimos este artículo citando una frase de Sthenhouse (1984: 285): "lo deseable en la innovación educativa no consiste en que perfeccionemos tácticas para hacer progresar muestra causa, sino en que mejoremos nuestra capacidad de someter a crítica nuestra práctica a la luz de nuestros conocimientos, y nuestros conocimientos a la luz de nuestra práctica".

\section{REFERENCIAS BIBLIOGRÁFICAS}

Altrichter, H., Posch, P. \& SomeKH, B. (1993). Teachers Investigate their Work. An introduction to the methods of action research, Londres: Routledge. 
BARTOlOME PinA, M. (1992). Investigación cualitativa en educación: ¿comprender o trasformar. Revista de Investigación Educativa, 20, 7 - 36.

Buendía Eisman, L. \& SALmerón Pérez, H. (1994). Intervención cooperativa a través de la investigación cooperativa. Revista de Investigación Educativa, 23, 226 - 231.

CARR, W. \& Kemmis, S. (1988). Teoría crítica de la enseñanza. La investigación-acción en la formación del profesorado. Barcelona: Martínez Roca.

Colas Bravo, Mã . P. (1994). La investigación - acción. En Colás, E. \& Buendía, L. (391 - 315). Investigación Educativa. Sevilla: Alfar.

Contreras Domingo, J. (1994). ¿Qué es?. Cuadernos de Pedagogía, 224, 8 - 14.

DESROCHE, H. (1981). La recherche coopérative comme recherche - action. En Actes u Colloque recherte action, Chicoutimi, UQAC (9-48), octubre 1981.

ELLIOTT, J. (1993). El cambio educativo desde la investigación-acción, Madrid: Morata.

Goyette,G. \& Lessard - Hérbert, M. (1988). La investigación - acción. Funciones, fundamentos e instrumentación. Barcelona: Alertes.

GRUNDY, S. (1982). Three modes of action research. En Kemmis, S. y McTaggart, R. (ed.)( 353 - 364): The Action Research Reader (3 ed .), Victoria: Deakin University.

KEMMIS, S. \& MCTAGGART, R. (1988). Cómo planificar la investigación-acción, Barcelona: Laertes.

LAPUeBLA, A. (1988). Una investigación colectiva. Cuadernos de Pedagogía, 157, 54 - 59.

LEWIN, K. (1973). Action research and minority problems. En K. Lewin (201 - 216): Resolving Social Coflicts: Selected Papers on Group Dynamics (ed. G. Lewin). London: Souvenir Press.

LÓPEZ GÓRRIZ, I. (1993). La investigación - acción como metodología de teorización y formación del profesor desde su práctica. Revista de Investigación Educativa, 71 - 92.

Perelló Pérez, R. Me, Membrive Marín, P. \& Vives Vítores, G. (1995). ¡A merendarj. Cuadernos de Pedagogía, 195, 48 - 50.

Pérez SeRrano, G. (1994). Investigación cualitativa. Retos e interrogantes I. Métodos. Madrid: La Muralla.

Rincón IGEA, D. \& Rincón IGEA, B. (2000). Revisión, planificación y aplicación de mejoras. Revista Interuniversitaria del Profesorado, 39, 51 - 73.

RINCÓN IGEA, D. (1997). Investigación acción - cooperativa. En MJ. Gregorio Rodríguez (71 - 97):Memorias del seminario de investigación en la escuela. Santa fe de Bogota 9 y 10 de Diciembre de 1997. Santa fe e Bogota: Quebecor Impreandes.

STENHOUSE, L. (1984). Investigación y desarrollo del currículo, Madrid: Morata.

WINTER, R. (1989). Learning from experience, Londres: Falmer Press. 


\title{
Contactar
}

Revista lberoamericana de Educación

\author{
Principal OEI
}

